\title{
Cognitive Control of Irrelevant Stimulus Changes
}

\author{
DOI: $10.17691 / \mathrm{stm} 2019.11 .1 .07$
}

Received October 17, 2018

M.V. Allakhverdov, PhD, Assistant Professor, Department of the Problems of Natural

and Humanitarian Sciences Convergence ${ }^{1}$;

T. Scott, PhD, Adj. Assistant Professor, Department of Psychology2;

A.S. Chernaya, Student';

V.M. Allakhverdov, DSc, Professor, Department of General Psycology ${ }^{1}$

${ }^{1}$ Saint Petersburg State University, 7/9 Universitetskaya Naberezhnaya, Saint Petersburg, 199034, Russia;

${ }^{2}$ College of Staten Island, The City University of New York, 2800 Victory Blvd, Staten Island, NY 10314, USA

It is known that psychological effects by suggestion, autosuggestion, context change, etc., can positively influence the individual's psychological mood and health. However, the mechanisms of this impact have not been studied so far. The problem is that a psychological impact must be perceived, but a consciousness which perceives information is not able to influence the physiological processes directly.

The aim of the study was to show that a psychological impact is possible by means of the mechanisms of cognitive control which automatically checks the correctness of the execution of the tasks assigned to the consciousness (or commands given to the consciousness) and the correctness of realizing concrete operations necessary for solving a task, and to confirm experimentally the existence of such involuntary cognitive control.

Materials and Methods. The present experimental investigation was based on the modified interference task, "Picture-Picture" test, in which images of two objects are simultaneously presented to the participants, and they should identify one target image, ignoring the second. Seventy-five people aged 18-29 years (75\% of women) participated in the confirmatory study. The tested subjects were to identify the object in the picture. The stimulus material also featured some irrelevant characteristics: a solid or dotted frame in which the target object was depicted.

Results. The data of the experiment have shown that regardless of the stimulus presentation order the task in the dotted frame are fulfilled longer than in the solid one $(p<0.001)$. However, in the post-experimental interview, the subjects said that the frame had not influenced their work in any way and they had not paid any attention to it. Thus, the results obtained show that the irrelevant parameters of the object (a frame in our case) are an essential part of defining the context in which the problem is being solved. Depending on this context the processes of cognitive control verify the execution of various tasks changing the time of the response.

Conclusion. It has been established that there exist cognitive control mechanisms that verify which task is being solved by a person. It has also been shown that the initiation of such verification automatically leads to susceptibility to psychological impact and physiological changes. At the same time, this control is noted to be triggered after the task (or command) has been apperceived.

Key words: cognitive control; interference effect; irrelevant stimulus characteristics; psychological impact.

\section{Introduction}

There is extensive literature about the effect of psychological impact on physiological processes. Suggestion and autosuggestion, placebo effect, changes of situation context, psychological mood affect the state regulation, efficiency of activity, and human health. For example, Blasi et al. [1] have carried out a meta-analysis of cognitive and emotional factors of doctor-patient interaction in which they showed that formation of a positive context of this interaction influences the efficiency of the treatment outcome. There is some paradoxicality in such capabilities of a psychological effect. Its impact is possible if there is awareness of the information (a person must hear a suggestive command, perceive the context change, understand the task set before him or her, etc.), however, consciousness is not able to control physiological processes directly.

Moreover, a psychological impact may occur even against one's conscious wish. In other words, even when one wishes to stop the process of the impact but cannot change physiological processes, their consciousness plays a crucial role in their susceptibility to suggestion. Let us illustrate it by an example. Let a person receive a command: "Your right arm is getting warm". Usually, after this command the arm is getting warm but only if a person has heard and perceived this command. The arm becomes warmer even if the person does not undertake any conscious efforts. We believe that there must exist a mechanism which automatically triggers when one gets the task (receives the command) and which is able, at least partially, to control physiological processes.

Corresponding author: Mikhail V. Allakhverdov, e-mail: m.allakhverdov@smolny.org 
The study aimed to describe the logic of this mechanism action.

\section{Materials and Methods}

Hypothesis. Individuals solving some task (executing the command given to them) must verify whether they are correctly executing the particular operations necessary for the solution of the task (executing the command). At the same time, they must check many other things, for example, whether they are solving this particular task. Are their actions in compliance with the expectations of the surrounding people (e.g. of the experimenter)? How are their actions agree with their own self-image (consistency check), and so on [2]? We assume that after the perception of the assigned task (command), this control activates automatically. The execution of the control operations is not usually perceived, but their result can be well perceived.

Returning to our example, let us assume that having received the command "Your right arm is getting warm", a person automatically checks whether his right arm warms up. For this purpose, the mechanism of cognitive control must collate the arm temperature with a reference standard of a warmer arm. However, to create such a standard, it is necessary to increase blood supply to the arm. As a result, the arm does warm up. For this explanation to be plausible, one must prove that a person verifies the correctness of executing the given commands. It is rather difficult to prove it from widespread phenomena of psychological impact. Baars has once elegantly written: "... there has been a very unfortunate tendency to focus on the most difficult and problematic cases, rather than on the simplest and most revealing ones... Trying to tackle the most difficult phenomena first is simply destructive of the normal process of science. It leads to confusion and controversy, rather than clarity" [3]. As we believe that the mechanisms of cognitive control are identical at any level, we try to prove the suggested hypothesis using simple cognitive tasks.

The main methodological approach. The easiest way to show the automaticity of cognitive control is to give an example of a situation when a given command (or an assigned task) requires, in an apparent or implicit form, to ignore something (think about nothing, pay no attention to anything, and so on). It would seem that it is easy to do nothing. However, these tasks are difficult to fulfill. We can explain it in the following way: as soon as a person attempts not to think about something, nevertheless they automatically verify whether they are thinking about it, and the ignored object penetrates the consciousness. This is what Wegner calls ironical thinking [4].

Let us consider the Stroop's phenomenon [5]. The task is to name the print color of the written words (the primary task) without reading the presented words (let it be the ignored task). The participants experience difficulty in executing this instruction. Why? Scientists commonly explain as the competition between the processes of reading the word and naming the color for a limited time resource. However, where does the competition originate from if reading is not required? We explain it differently. The emergence of Stroopinterference is a consequence of the control of the ignoring task. As soon as subjects start checking whether they are reading the words, they automatically read them. This is confirmed by the decrease of interference when the main task is complicated (using color tints, using two colors for writing the words, additional tasks in the form of an endless monolog, or even presence of other people) [6]. If not for the ad hoc hypotheses, interpretation using the idea of limited resources implies the opposite results. We suggest the following interpretation: the more complicated is the main task, the less controlled is the "task to ignore".

We have tested the suggested interpretation using the "Picture-picture interference" experimental paradigm in which subjects are presented with two images, one of which should be named as quickly as possible while ignoring the other. The effect of interference in this variant has been shown in previous studies [7]. However, despite its seeming similarity with Stroop phenomenon, some authors believe that these two phenomena have different mechanisms of action [8]. This point of view came to be because the currently existing popular interpretations of Stroop interference are inadequate to explain the delayed response in the "Picture-Picture" test. However, if we presuppose the mechanisms of cognitive task control, these two effects should involve the same cognitive processes.

In our experiment participants had to identify the object in the target picture which is presented inside the other picture (ignored distractor). Both pictures represented the contours of the images referred to the categories of "fruits" or "vegetables" (Figure 1). The contour in both pictures was solid (condition 1), or the target object had a solid contour while the distractor was represented by a dotted line (condition 2), or the target had a dotted outline and the distractor a solid one (condition 3).

Each person performed the assignment in all three conditions and went through a preliminary learning

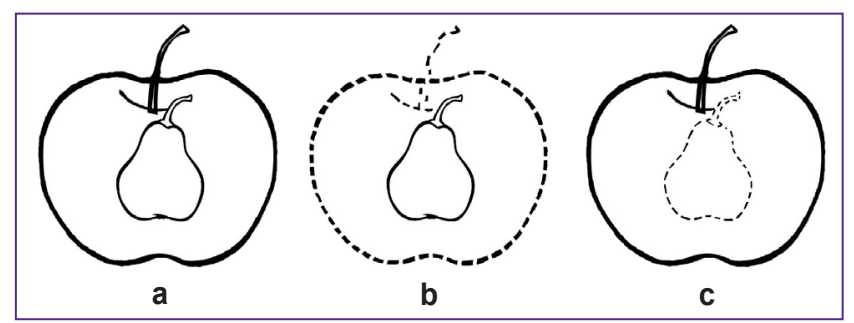

Figure 1. Modifications of stimulus material in the "Picture-Picture" paradigm:

(a) condition 1; (b) condition 2; (c) condition 3 
series. The results showed that the interference effect observed in all conditions (for details look in the study [9]). We have proposed that identification of the dotted target is a more difficult task than the identification of the target drawn with a solid line. When the picture is in a solid frame, a person considers the frame as a background and reduces control over whether one chose the figure and background correctly. The picture in the dotted frame requires the control: whether the object is identified correctly. If so, the interference must be the least of all when the target is presented in a dotted frame. It was just the case in the research. Is it true that presenting the picture in the dotted frame serves as a complication of the primary assignment? To validate this, we carried out the following investigation.

Participants. Seventy-five people aged from 18 to 29 years (75\% of women) took part in the investigation. The participants were randomly divided into three equal groups which differed by order of stimuli presentation. The study complies with the Declaration of Helsenki (2013) and is approved by the Ethics Committee of the Saint Petersburg State University. Informed consent was obtained from each patient.

Study design. In our experiment, we used a twofactor design with one between-group factor "the type of irrelevant characteristics" with two levels (dotted and solid frame) and one within-group factor "the order of stimulus presentation" with three levels.

Stimulus material. Four black-and-white (with grey tints) images of an apple, pear, grape, raspberry were used as stimuli. At the preliminary stage, these images were presented to a group of experts (10 people) who accurately identified the depicted objects. At the experimental stage, the objects were presented in a black-and-white rectangular solid or dotted-line frame (Figure 2 (a), (b)). In the training series, the fruit images were presented on a white background without a frame (Figure 2 (c)).

The experiment was programmed in PsychoPy 1.85.6. All images were shown on the display of the laptop Apple 13" MacBook Air notebook computer (with $1440 \times 900$ resolution).

Procedure. During the experiment, the task of the experiment was to identify as quick and accurate

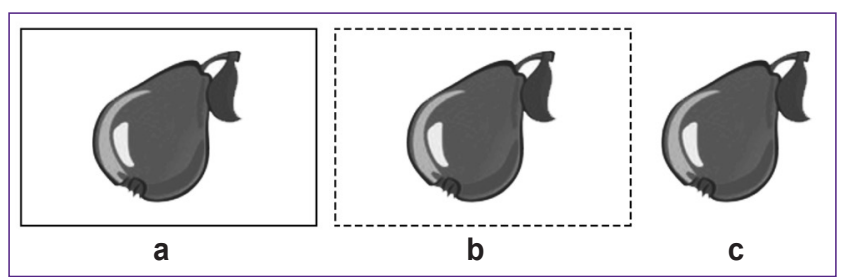

Figure 2. Examples of the stimulus material used in the experiment:

(a) a target object in a solid frame; (b) a target object in a dotted frame; (c) a target object without a frame (learning stage) as possible the object presented to the participants by pressing the key on the keyboard with the images stick to them: q corresponded to the picture of a grape; $\mathrm{s}$ - apple; $\mathrm{k}$ - pear; $\mathrm{p}$ - raspberry. These keys were chosen because they were situated at a distance large enough to prevent an unwanted keystroke, on the one hand, and they allow the fingers to be placed conveniently in order to react quickly to the stimuli, on the other. To get familiar with the pictures and to get used to the keys position, the participants went through the training phase consisting of 24 presentations ( 6 series of 4 images without a frame).

Once the participant has adapted to the experiment, the main stage of the experiment began. Depending on the group the participants were presented with 96 stimuli in the following order:

group 1: first, a block of stimuli in a solid frame (48 stimuli -12 series of 4 stimuli) followed by a block of stimuli in a dotted frame (48 stimuli -12 series of 4 stimuli);

group 2: first, a block of stimuli in a dotted frame, followed by a block of stimuli in a solid frame (the number of stimuli corresponding to that in group 1);

group 3: stimuli with a random order of the frame contour (48 stimuli for each frame type).

From the instruction, the participants knew that the target images would be in a frame, but they were unaware that the frame would have different contours.

\section{Results}

Training stage. The learning stage analysis showed that the participants performed the task with an average accuracy of $97 \%$ in all groups, i.e., they coped with the assigned tasks and quickly identified the presented images. Since at the learning stage the participants only familiarized themselves with the experimental process and learned the location of the appropriate keys on a keyboard, the time of the task execution appeared to be much longer than at the main stage $(F(1,72)=48.587$; $p<0.001)^{1}$ regardless of the group $(p>0.5)$ (Table 1$)$. The results presented allowed us to suppose that the task execution time in all the groups does not differ significantly ( $p>0.8)$.

As this stage was considered preliminary and the assignments were slightly different, further comparison with the main stage seemed to be of no value, and therefore this part of the experiment was excluded from further analysis.

Main stage. As in the case with the learning series, the participants successfully coped with the given assignment. There was no significant difference between

${ }^{1}$ Hereinafter, when Fisher coefficient is greater than 1 , values of Fisher criterion (F) are given indicating in brackets degrees of freedom for intergroup and intragroup dispersion, respectively, and the resulted level of significance. Otherwise only the level of significance is presented. 
Table 1

An average time of response to the stimuli (ms) in the groups at the learning and experimental stages, $\mathrm{M} \pm \mathrm{SE}$

\begin{tabular}{llll}
\hline \multicolumn{1}{r}{ Stages } & Group 1 & Group 2 & Group 3 \\
\hline Training & $946 \pm 39$ & $956 \pm 51$ & $1005 \pm 42$ \\
Main & $860 \pm 36$ & $850 \pm 36$ & $890 \pm 37$ \\
\hline
\end{tabular}

the groups in the number of mistakes made $(p>0.8)$; the average accuracy of the responses was high (98\%). A large number of correct responses are evidence that the task itself was regarded as manageable, and caused no additional difficulties for participants, and the mistakes they made occurred at random. The differences in the accuracy of responses for both types of the frame in which the object was presented were also insignificant $(p>0.5)$ (Table 2). The time of the task execution was also insignificant for different groups $(p>0.5)$ and did not depend on the interaction of the presentation order factor and the type of the contour $(F(2,72)=1.398 ; p=0.253)$. However, it took longer to fulfill the assignment in the dotted frame than in the solid in all groups regardless of the order of stimulus presentation $(F(1,72)=5.73$; $\mathrm{p}=0.019$ ).

Thus, the results obtained give evidence in support of the suggested hypothesis: using fragmented frame contour in which the target is depicted (entirely unrelated to the execution of the target assignment) increases the time it takes participants to perform the task. The longer latency, in this case, is the result we expected based on the interpretation of the previous experiment in the "Picture-Picture" paradigm.

There is one more evidence for the validity of this theory. At the main stage, we can also observe learning. Results of participants from the third group clearly showed the learning process: they identified 48 stimuli in the second half of the experiment much quicker than the first 48 stimuli (there were no differences between the stimuli in the first and second halves). Thus, the execution of the first part of the experiment took $921 \pm 39 \mathrm{~ms}$ while the second took only $858 \pm 35 \mathrm{~ms}$ $(t(24)=5.346 ; p<0.001)^{2}$.

During learning performance of operations becomes automatic, i.e., the control over them decreases. In the case of the experiment, we have two operations: identification of the object from the background and target object naming. We observed no learning in the results of group 1 (i.e., the first half of the experiment the image is in the solid frame, the second - in the dotted frame). In contrast, the participants of group 2 (the first half in the dotted frame, the second in the solid frame) demonstrated the quickest identification of the

\footnotetext{
${ }^{2}$ Hereinafter the values of Student's t-test for related samples are given indicating in brackets degrees of freedom and the level of significance.
}

Table 2

Average response time ( $\mathrm{ms}$ ) in the groups depending on the type of stimulus presented at the main stage, $\mathrm{M} \pm \mathrm{SE}$

\begin{tabular}{llll}
\hline \multicolumn{1}{c}{ Contour type } & Group 1 & Group 2 & Group 3 \\
\hline Dotted frame & $866 \pm 39$ & $881 \pm 33$ & $900 \pm 38$ \\
Solid frame & $855 \pm 36$ & $819 \pm 43$ & $879 \pm 36$ \\
\hline
\end{tabular}

object in the second half $(t(24)=2.294 ; p=0.031)$. Since object identification is similar in all cases, the differences in learning in groups 1 and 2 are likely to be explained by the change in the control over figure-background relations. Since the identification of the object in the dotted frame is a more complicated process, the transition to a more straightforward task, when object presented in the solid frame, leads to a substantial decrease of control over this particular operation.

\section{Discussion}

The research shows that our assumption about the processes of cognitive control has experimental verification. The control usually happens unconsciously as many participants in their post-experimental interview stated the belief that the frame had no influence on their result and that they did not pay any attention to it. However, the process of identification of the target object from the background does depend on the type of the frame. We are accustomed to the fact that frames are everywhere (e.g., in computers, pictures, TV-sets), and therefore, even unconsciously, we rarely control the correctness of object identification from the background when we see a picture in a solid frame.

We have established that cognitive control is triggered unconsciously. Let us return to the logic of this control work which leads to the emergence of psychological impacts on the organism state. We will try to describe how the placebo effect originates. A doctor gives a tablet which, as the patient consciously supposes, will help him or her. Cognitive control is triggered automatically: does the organism feel better? The present state of the organism must be compared with a model of a healthier state, and the very process of building such model results in health improvement. The same algorithm explains the reverse process of nocebo since in this case, a patient apprehends the appearance of sideeffects.

The fact that it is the cognitive control that plays a significant role in a psychological effect can be seen by an example of the psychotherapeutic technique of paradoxical intention, developed by V. Frankl. In the situation when a surgeon is afraid that her hands will be shaking during the operation or a child suffering from enuresis is afraid to make the bed wet Frankl suggests giving a command permitting the execution of those actions which a person fears. For example, the 
surgeon is told to make her hands shake much more intensively. Such instruction helps put an end to tremor in her hands. In our studies, we have shown that if a person is persistently making the same mistake in some sensorimotor skill (e.g., making the same misprint in the word typing it on the computer), this mistake may disappear if it is consciously made several times. Why may paradoxical intention be so productive? We asserted that cognitive control is triggered unconsciously but only after the comprehension of the task. A surgeon has a task: the hands must not shake. Paradoxical intention eliminates the task, and cognitive control is not triggered.

Our investigation has also demonstrated the significance of context changes for the processes regulating cognitive control. For instance, in the studies of Zaporozhets [10], people recovering after a physical trauma could not move their thumb and index finger more than 10-15 $\mathrm{mm}$ apart when they were instructed to expand them as far as possible. However, when they were asked to take an object with a much greater width, they easily completed this task. When a person is instructed to do something at a one's maximum, they must inwardly define a certain limit which they form based on their experience and implicit notions about their capabilities. Preservation of this limit becomes a complementary task and, naturally, its execution needs to be controlled. When the task changes, and consequently, the situation context changes too, cognitive control switches to the fulfillment of another task enabling a person to perform the given task at a higher level.

\section{Conclusion}

The results of the present research demonstrated on simple cognitive tasks that the processes of cognitive task control regulate human activity. Here we discuss the importance of these controlling processes in case of a psychological impact. Undoubtedly the research cannot uncover all causes and reasons of psychological impacts but encourage the researchers to analyze these reasons taking into consideration the work of cognitive control mechanisms.

Study funding. The work was supported by the Russian Foundation for Basic Research (grant No.18013-01212).

Conflicts of interest. The authors declare no conflicts of interest related to this study.

\section{References}

1. Blasi Z.D., Harkness E., Ernst E., Georgiou A., Kleijnen J. Influence of context effects on health outcomes: a systematic review. Lancet 2001; 357(9258): 757-762, https:// doi.org/10.1016/s0140-6736(00)04169-6.

2. Allakhverdov V.M. The psyche and consciousness in the logic of cognition. Vestnik Sankt-Peterburgskogo universiteta. Seriya 16. Psikhologiya. Pedagogika 2016; 1: 35-46.

3. Baars B.A. Cognitive theory of consciousness. Cambridge: Cambridge University Press; 1995.

4. Wegner D.M. Ironic processes of mental control. Psychol Rev 1994; 101(1): 34-52, https://doi. org/10.1037/0033-295x.101.1.34.

5. Stroop J.R. Studies of interference in serial verbal reactions. J Exp Psychol Gen 1992; 121(1): 15-23, http:// dx.doi.org/10.1037/0096-3445.121.1.15.

6. Allakhverdov V.M., Allakhverdov M.V. Stroop effect: interference as a logic paradox. Vestnik Sankt-Peterburgskogo universiteta. Seriya 16. Psikhologiya. Pedagogika 2014; 4: 90-102.

7. Glaser W.R., Glaser M.O. Context effects in Stroop-like word and picture processing. J Exp Psychol Gen 1989; 118(1): 13-42, https://doi.org/10.1037//0096-3445.118.1.13.

8. Damian M.F., Bowers J.S. Locus of semantic interference in picture-word interference tasks. Psychon Bull Rev 2003; 10(1): 111-117, https://doi.org/10.3758/ bf03196474.

9. L'vova O.V., Allakhverdov M.V. On the task control in the "drawing-drawing" test. Psikhologicheskie issledovaniya 2018; 9: 33-42.

10. Zaporozhets A.V. Razvitie proizvol'nykh dvizheniy [Development of voluntary movements]. Moscow; 1960. 